\title{
A geometric model for cluster categories of type $D_{n}$
}

\author{
Ralf Schiffler
}

Received: 30 August 2006 / Accepted: 27 March 2007 /

Published online: 26 April 2007

(C) Springer Science+Business Media, LLC 2007

\begin{abstract}
We give a geometric realization of cluster categories of type $D_{n}$ using a polygon with $n$ vertices and one puncture in its center as a model. In this realization, the indecomposable objects of the cluster category correspond to certain homotopy classes of paths between two vertices.
\end{abstract}

Keywords Cluster category · Triangulated surface $\cdot$ Punctured polygon · Elementary move

\section{Introduction}

Cluster categories were introduced in [6] and, independently, in [11] for type $A_{n}$, as a means for better understanding of the cluster algebras of Fomin and Zelevinsky $[15,16]$. Since then cluster categories have been the subject of many investigations; see, for instance, [1, 2, 7-10, 12-14, 21-23, 26].

In the approach of [6], the cluster category $\mathcal{C}_{A}$ is defined as the quotient $\mathcal{D}^{b} A / F$ of the derived category $\mathcal{D}^{b} A$ of a hereditary algebra $A$ by the endofunctor $F=\tau_{\mathcal{D}^{b} A}^{-1}$ [1], where $\tau_{\mathcal{D}^{b} A}$ is the Auslander-Reiten translation, and [1] is the shift. On the other hand, in the approach of [11], which is only valid in type $A_{n}$, the cluster category is realized by an ad-hoc method as a category of diagonals of a regular polygon with $n+3$ vertices. The morphisms between diagonals are constructed geometrically using so-called elementary moves and mesh relations. In that realization, clusters are in one-to-one correspondence with triangulations of the polygon, and mutations are given by flips of diagonals in the triangulation. Recently, Baur and Marsh [5] have generalized this model to $m$-cluster categories of type $A_{n}$.

R. Schiffler $(\bowtie)$

Department of Mathematics and Statistics, University of Massachusetts at Amherst, Amherst, MA 01003-9305, USA

e-mail: schiffler@math.umass.edu 
In this paper, we give a geometric realization of the cluster categories of type $D_{n}$ in the spirit of [11]. The polygon with $(n+3)$ vertices has to be replaced by a polygon with $n$ vertices and one puncture in the center, and instead of looking at diagonals, which are straight lines between two vertices, one has to consider homotopy classes of paths between two vertices, which we will call edges. This punctured polygon model has appeared recently in the work of Fomin, Shapiro, and Thurston [17] on the relation between cluster algebras and triangulated surfaces. Let us point out that they work in a vastly more general context, and the punctured polygon is only one example of their theory. We define the cluster category by an ad-hoc method as the category of (tagged) edges inside the punctured polygon. Morphisms are defined using so-called elementary moves and mesh relations, which are generalizations of the elementary moves and mesh relations of [11]. Our main results are the equivalence of the category of tagged edges and the cluster category of [6], see Theorem 4.3, and the realization of the dimension of $\mathrm{Ext}^{1}$ of tagged edges as the number of crossings between the same tagged edges, see Theorem 5.3.

The article is organized as follows. After a brief preliminary section, in which we fix the notation and recall some concepts needed later, Sect. 3 is devoted to the definition of the category $\mathcal{C}$ of tagged edges. In Sect. 4, we show the equivalence of this category and the cluster category and, in Sect. 5, we study Ext ${ }^{1}$ of indecomposable objects in $\mathcal{C}$. As an application, in Sect. 6, we describe Auslander-Reiten triangles, tilting objects and exchange relations and show a geometric method to construct the Auslander-Reiten quiver of any cluster tilted algebra of type $D_{n}$, using a result of [7].

\section{Preliminaries}

\subsection{Notation}

Let $k$ be an algebraically closed field. If $Q$ is a quiver, we denote by $Q_{0}$ the set of vertices and by $Q_{1}$ the set of arrows of $Q$. The path algebra of $Q$ over $k$ is denoted by $k Q$. It is of finite representation type if there is only a finite number of isoclasses of indecomposable modules. By Gabriel's theorem $k Q$ is of finite representation type if and only if $Q$ is a Dynkin quiver, that is, the underlying graph of $Q$ is a Dynkin diagram of type $A_{n}, D_{n}$, or $E_{n}[18]$.

If $A$ is an algebra, we denote by $\bmod A$ the category of finitely generated right $A$-modules and by ind $A$ the full subcategory whose objects are a full set of representatives of the isoclasses of indecomposable $A$-modules. Let $\mathcal{D}^{b} A=\mathcal{D}^{b}(\bmod A)$ denote the derived category of bounded complexes of finitely generated $A$-modules. For further facts about $\bmod (A)$ and $\mathcal{D}^{b} A$, we refer the reader to $[3,4,19,25]$.

If $\mathcal{A}$ is an additive $k$-category, then its additive hull $\oplus \mathcal{A}$ is defined as follows: the objects of $\oplus \mathcal{A}$ are direct sums of objects in $\mathcal{A}$, morphisms $\oplus_{i} X_{i} \rightarrow \oplus_{j} Y_{j}$ are given componentwise by morphisms $X_{i} \rightarrow Y_{j}$ of $\mathcal{A}$, and the composition of morphisms is given by matrix multiplication.

\subsection{Translation quivers}

Following [24], we define a stable translation quiver $(\Gamma, \tau)$ to be a quiver $\Gamma=$ $\left(\Gamma_{0}, \Gamma_{1}\right)$ without loops together with a bijection $\tau$ (the translation) such that the 
number of arrows from $y \rightarrow x$ is equal to the number of arrows from $\tau x \rightarrow y$ for any $x, y \in \Gamma_{0}$. Given a stable translation quiver $(\Gamma, \tau)$, a polarization of $\Gamma$ is a bijection $\sigma: \Gamma_{1} \rightarrow \Gamma_{1}$ such that $\sigma(\alpha): \tau x \rightarrow y$ for every arrow $\alpha: y \rightarrow x \in \Gamma_{1}$. If $\Gamma$ has no multiple arrows, then there is a unique polarization.

Given a quiver $Q$, one can construct a stable translation quiver $\mathbb{Z} Q$ as follows: $(\mathbb{Z} Q)_{0}=\mathbb{Z} \times Q_{0}$; the number of arrows in $Q$ from $(i, x)$ to $(j, y)$ equals the number of arrows in $Q$ from $x$ to $y$ if $i=j$ and equals the number of arrows in $Q$ from $y$ to $x$ if $j=i+1$, and there are no arrows otherwise. The translation $\tau$ is defined by $\tau((i, x))=(i-1, x)$.

The path category of $(\Gamma, \tau)$ is the category whose objects are the vertices of $\Gamma$, and given $x, y \in \Gamma_{0}$, the $k$-space of morphisms from $x$ to $y$ is given by the $k$-vector space with basis the set of all paths from $x$ to $y$. The composition of morphisms is induced from the usual composition of paths.

The mesh ideal in the path category of $\Gamma$ is the ideal generated by the mesh relations

$$
m_{x}=\sum_{\alpha: y \rightarrow x} \sigma(\alpha) \alpha
$$

The mesh category $\mathcal{M}(\Gamma, \tau)$ of $(\Gamma, \tau)$ is the quotient of the path category of $(\Gamma, \tau)$ by the mesh ideal. In general, the mesh category depends on the polarization. In this article, however, we only consider mesh categories with unique polarizations.

Important examples of translation quivers are the Auslander-Reiten quivers of the derived categories of hereditary algebras of finite representation type. We shall need the following proposition.

\section{Proposition 2.1 Let $Q$ be a Dynkin quiver. Then}

1. For any quiver $Q^{\prime}$ of the same Dynkin type as $Q$, the derived categories $\mathcal{D}^{b} k Q$ and $\mathcal{D}^{b} k Q^{\prime}$ are equivalent.

2. The Auslander-Reiten quiver of $\mathcal{D}^{b} k Q$ is $\mathbb{Z} Q$.

3. The category ind $\mathcal{D}^{b} k Q$ is equivalent to the mesh category of $\mathbb{Z} Q$.

Proof See [20, I.5].

\subsection{Cluster categories}

Let $\mathcal{C}_{D_{n}}$ be the cluster category of type $D_{n}$, see [6]. By definition, $\mathcal{C}_{D_{n}}$ is the quotient of the derived category $\mathcal{D}^{b} A$ of a hereditary algebra $A$ of type $D_{n}$ by the endofunctor $F=\tau_{\mathcal{D}^{b} A}^{-1}$ [1], where $\tau_{\mathcal{D}^{b} A}$ is the Auslander-Reiten translation in $\mathcal{D}^{b} A$, and [1] is the shift. Thus the objects $\tilde{M}$ of $\mathcal{C}_{D_{n}}$ are the orbits $\tilde{M}=\left(F^{i} M\right)_{i \in \mathbb{Z}}$ of objects $M \in \mathcal{D}^{b} A$ and $\operatorname{Hom}_{\mathcal{C}_{D_{n}}}(\tilde{M}, \tilde{N})=\bigoplus_{i \in \mathbb{Z}} \operatorname{Hom}_{\mathcal{D}^{b} A}\left(M, F^{i} N\right)$. Let us denote the vertices of the 
Dynkin diagram of $D_{n}$ as follows:

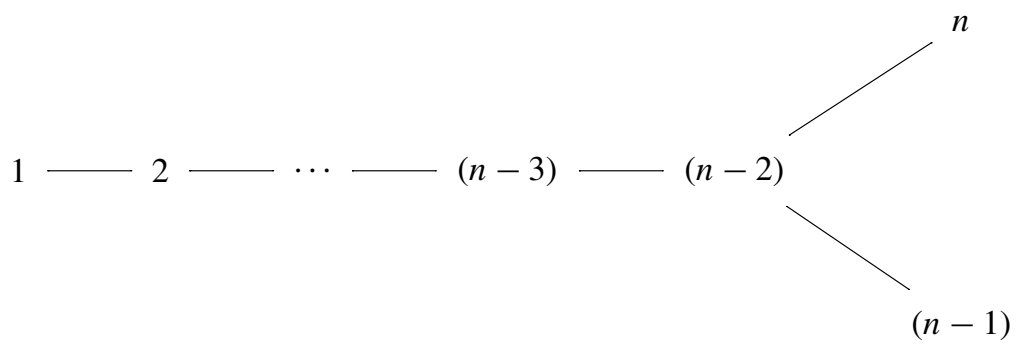

and, for convenience, let us choose the algebra $A$ to be the path algebra $k Q$ of the quiver

$$
Q=1 \rightarrow 2 \rightarrow \cdots \rightarrow(n-3) \rightarrow(n-2) \longrightarrow_{(n-1) .}^{n}
$$

Since, by Proposition 2.1, the Auslander-Reiten quiver of $\mathcal{D}^{b} A$ is the stable translation quiver $\mathbb{Z} Q$, the labels of $Q_{0}$ induce labels on the vertices of $\mathbb{Z} Q$ as usual (see Fig. 1):

$$
(\mathbb{Z} Q)_{0}=\left\{(i, j) \mid i \in \mathbb{Z}, j \in Q_{0}\right\}=\mathbb{Z} \times\{1, \ldots, n\} .
$$

Moreover, we can identify the indecomposable objects of $\mathcal{D}^{b} A$ with the vertices of $\mathbb{Z} Q$. Let $P_{1}$ be the indecomposable projective module corresponding to the vertex $1 \in Q_{0}$. Then, by defining the position of $P_{1}$ to be $(1,1)$, we have a bijection

$$
\text { pos : ind } \mathcal{D}^{b} A \rightarrow \mathbb{Z} \times\{1, \ldots, n\} .
$$

In other terms, for $M \in$ ind $\mathcal{D}^{b} A$, we have $\operatorname{pos}(M)=(i, j)$ if and only if $M=$ $\tau_{\mathcal{D}^{b} A}^{-i} P_{j}$, where $P_{j}$ is the indecomposable projective $A$-module at vertex $j$. The integer $j \in\{1, \ldots, n\}$ is called the level of $M$ and is denoted by level $(M)$.

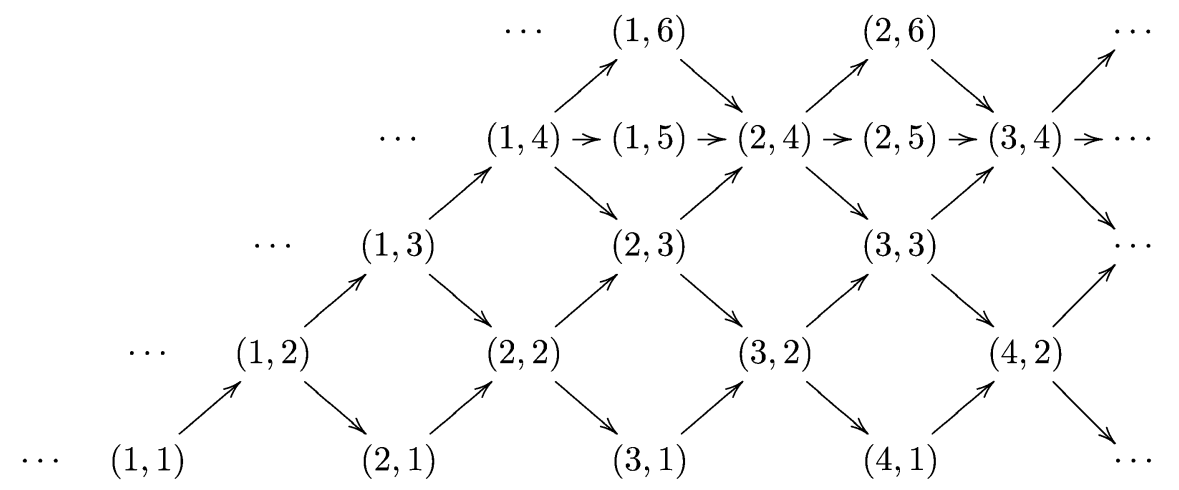

Fig. 1 Labels of the Auslander-Reiten quiver of $\mathcal{D}^{b} A$ if $n=6$ 
There is a quiver automorphism $\mathbb{Z} Q \rightarrow \mathbb{Z} Q$ that fixes all indecomposable objects $M$ with level $(M)<n-1$ and exchanges the indecomposable objects at positions $(i, n-1)$ and $(i, n)$ for all $i \in \mathbb{Z}$. If $M$ is an indecomposable object with level $(M) \geq n-1$, then let $M^{-}$be its image under this quiver automorphism. The structure of the module category of $A$ and of its derived category is well known. In particular, we have the following result.

Lemma 2.2 Let $M \in \operatorname{ind} \mathcal{D}^{b} A$.

1. If $n$ is even, then $M[1]=\tau_{\mathcal{D}^{b} A}^{-n+1} M$.

2. If $n$ is odd, then

$$
M[1]= \begin{cases}\tau_{\mathcal{D}^{b} A}^{-n+1} M & \text { if level }(M) \leq n-2, \\ \tau_{\mathcal{D}^{b} A}^{-n+1} M^{-} & \text {if level }(M) \in\{n-1, n\} .\end{cases}
$$

Proof It suffices to prove the statement in the case where $M=P$ is an indecomposable projective $A$-module. Let $v_{A}$ denote the Nakayama functor of $\bmod A$. Then $P[1]=\tau_{\mathcal{D}^{b} A}^{-1} v_{A} P$. Now the statement follows from [19, Proposition 6.5].

We also define the position of indecomposable objects of the cluster category $\mathcal{C}_{A}=$ $\mathcal{D}^{b} A / F$. The set $\bmod A \sqcup A[1]$ is a fundamental domain for the cluster category (here $A[1]$ denotes the first shift of all indecomposable projective $A$-modules). Then, for $\tilde{X} \in \operatorname{ind} \mathcal{C}_{A}$, we define $\operatorname{pos}(\tilde{X})$ to be $\operatorname{pos}(X)$, where $X$ is the unique element in the fundamental domain such that $\tilde{X}$ is the orbit of $X$. Thus $\operatorname{pos}(\tilde{X}) \in\{1,2, \ldots, n\}^{2}$.

We recall now a well-known result that uses the position coordinates to describe the dimension of the space of morphisms between indecomposable $\mathcal{C}_{A}$-objects. Let $M, N \in \operatorname{ind} \mathcal{C}_{A}$ be such that $\operatorname{pos}(M)=(1, m)$ and $\operatorname{pos}(N)=(i, j)$. The next proposition follows from the structure of the mesh category.

Proposition 2.3 The dimension of $\operatorname{Hom}_{\mathcal{C}_{A}}(M, N)$ is 0,1 , or 2 and it can be characterized as follows:

1. If $m \leq n-2$, then $\operatorname{dim} \operatorname{Hom}_{\mathcal{C}_{A}}(M, N) \neq 0$ if and only if

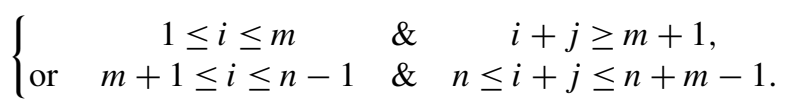

2. If $m \in\{n-1, n\}$, define $m^{\prime}$ to be the unique element in $\{n-1, n\} \backslash\{m\}$. Then $\operatorname{dim} \operatorname{Hom}_{\mathcal{C}_{A}}(M, N) \neq 0$ if and only if

$$
\left\{\begin{array}{cccccc} 
& 2 \leq i \leq n-1 & \& & i+j \geq n & \& & j \leq n-2, \\
\text { or } \quad 1 \leq i \leq n-1 & \& & j=m & \& & i \text { is odd } \\
\text { or } \quad 1 \leq i \leq n-1 & \& & j=m^{\prime} & \& & i \text { is even. }
\end{array}\right.
$$

Moreover, $\operatorname{dim} \operatorname{Hom}_{\mathcal{C}_{A}}(M, N)=2$ if and only if

$$
2 \leq m \leq n-2 \quad \& \quad 2 \leq i \leq m \quad \& \quad 2 \leq j \leq n-2 \quad \& \quad i+j \geq n .
$$


To illustrate this statement, we give an example where $n=6$ and $M$ is on position $(1,3)$. The following picture shows the Auslander-Reiten quiver of $\mathcal{C}_{A}$ (without arrows) and where the label on any vertex $N$ is $\operatorname{dim}_{\mathcal{H o m}_{\mathcal{A}}}(M, N)$. If this number is zero, we write a dot instead of 0 . The two underlined vertices should be identified, they indicate the position of $M$.

\begin{tabular}{|c|c|c|c|c|c|c|c|c|c|c|c|c|c|c|c|c|c|c|}
\hline . & & 1 & & 1 & & 1 & & . & & . & & & & & & & & \\
\hline . & 1 & 1 & 2 & 1 & 2 & 1 & 1 & . & . & & & & & & & & & \\
\hline$\underline{1}$ & & 1 & & 2 & & 1 & & 1 & & & & & & & & & & \\
\hline & 1 & & 1 & & 1 & & 1 & & & & & & & & & & & \\
\hline . & & 1 & & $\cdot$ & & 1 & & $\cdot$ & & . & & & & & & & & \\
\hline
\end{tabular}

\section{The category of tagged edges}

\subsection{Tagged edges}

Consider a regular polygon with $n \geq 3$ vertices and one puncture in its center.
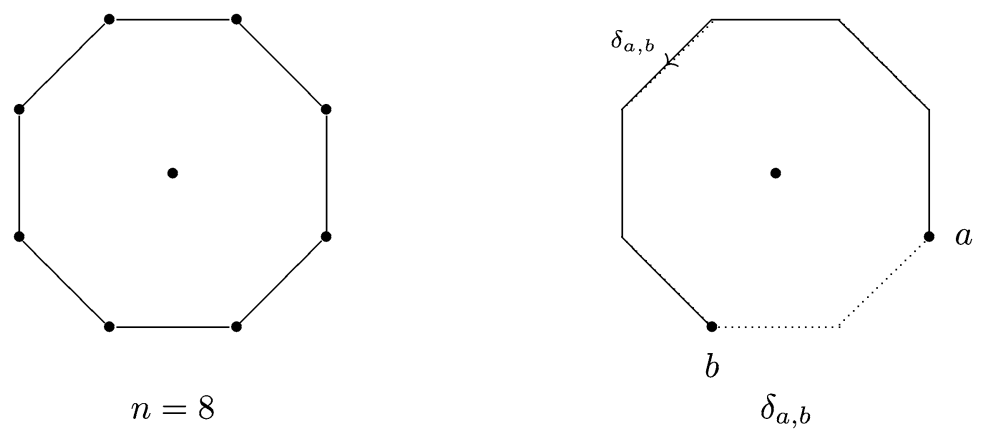

If $a \neq b$ are any two vertices on the boundary, then let $\delta_{a, b}$ denote a path along the boundary from $a$ to $b$ in counterclockwise direction which does not run through the same point twice. Let $\delta_{a, a}$ denote a path along the boundary from $a$ to $a$ in counterclockwise direction which goes around the polygon exactly once and such that $a$ is the only point through which $\delta_{a, a}$ runs twice. For $a \neq b$, let $\left|\delta_{a, b}\right|$ be the number of vertices on the path $\delta_{a, b}$ (including $a$ and $b$ ), and let $\left|\delta_{a, a}\right|=n+1$. Two vertices $a, b$ are called neighbors if $\left|\delta_{a, b}\right|=2$ or $\left|\delta_{a, b}\right|=n$, and $b$ is the counterclockwise neighbor of $a$ if $\left|\delta_{a, b}\right|=2$.

An $e d g e$ is a triple $(a, \alpha, b)$ where $a$ and $b$ are vertices of the punctured polygon and $\alpha$ is a path from $a$ to $b$ such that

(E1) $\alpha$ is homotopic to $\delta_{a, b}$.

(E2) Except for its starting point $a$ and its endpoint $b$, the path $\alpha$ lies in the interior of the punctured polygon.

(E3) $\alpha$ does not cross itself, that is, there is no point in the interior of the punctured polygon through which $\alpha$ runs twice.

(E4) $\left|\delta_{a, b}\right| \geq 3$. 
Note that condition (E1) implies that if $a=b$ then $\alpha$ is not homotopic to the constant path, and condition (E4) means that $b$ is not the counterclockwise neighbor of $a$.

Two edges $(a, \alpha, b),(c, \beta, d)$ are equivalent if $a=c, b=d$, and $\alpha$ is homotopic to $\beta$. Let $E$ be the set of equivalence classes of edges. Since the homotopy class of an edge is already fixed by condition (E1), an element of $E$ is uniquely determined by an ordered pair of vertices $(a, b)$. We will therefore use the notation $M_{a, b}$ for the equivalence class of edges $(a, \alpha, b)$ in $E$.

Define the set of tagged edges $E^{\prime}$ by

$$
E^{\prime}=\left\{M_{a, b}^{\epsilon} \mid M_{a, b} \in E, \epsilon= \pm 1 \text { and } \epsilon=1 \text { if } a \neq b\right\} .
$$

If $a \neq b$, we will often drop the exponent and write $M_{a, b}$ instead of $M_{a, b}^{1}$. In other terms, for any ordered pair $(a, b)$ of vertices with $a \neq b$ and $b$ not the counterclockwise neighbor of $a$, there is exactly one tagged edge $M_{a, b} \in E^{\prime}$, and, for any vertex $a$, there are exactly two tagged edges $M_{a, a}^{-1}$ and $M_{a, a}^{1}$. A simple count shows that there are $\frac{n !}{(n-2) !}-n+2 n=n^{2}$ elements in $E^{\prime}$. These tagged edges will correspond to the indecomposable objects in the cluster category.

Remark 3.1 Our definition of (tagged) edges is for the punctured polygon only. It is an adapted version of the much more general definitions of (tagged) arcs in [17].

Remark 3.2 In pictures, the tagged edges $M_{a, a}^{\epsilon}$ that start and end at the same vertex $a$ will not be represented as loops around the puncture but as lines from the vertex $a$ to the puncture. If $\epsilon=-1$, the line will have a tag on it and if $\epsilon=1$, there will be no tag. The reason for this is the definition of the crossing number in the next section.

\subsection{Crossing numbers}

Let $M=M_{a, b}^{\epsilon}$ and $N=M_{c, d}^{\epsilon^{\prime}}$ be in $E^{\prime}$, and let $\Delta^{0}$ denote the interior of the punctured polygon. The crossing number $e(M, N)$ of $M$ and $N$ is the minimal number of intersections of $M_{a, b}$ and $M_{c, d}$ in $\Delta^{0}$. More precisely,

1. If $a \neq b$ and $c \neq d$, then

$$
e(M, N)=\min \left\{\operatorname{Card}\left(\alpha \cap \beta \cap \Delta^{0}\right) \mid(a, \alpha, b) \in M_{a, b},(c, \beta, d) \in M_{c, d}\right\} .
$$

2. If $a=b$ and $c \neq d$, let $\alpha$ be the straight line from $a$ to the puncture. Then

$$
e(M, N)=\min \left\{\operatorname{Card}\left(\alpha \cap \beta \cap \Delta^{0}\right) \mid(c, \beta, d) \in M_{c, d}\right\} .
$$

3. If $a \neq b$ and $c=d$, let $\beta$ be the straight line from $c$ to the puncture. Then

$$
e(M, N)=\min \left\{\operatorname{Card}\left(\alpha \cap \beta \cap \Delta^{0}\right) \mid(a, \alpha, b) \in M_{a, b}\right\} .
$$

4. If $a=b$ and $c=d$, that is, $M=M_{a, a}^{\epsilon}$ and $N=M_{c, c}^{\epsilon^{\prime}}$, then

$$
e(M, N)= \begin{cases}1 & \text { if } a \neq c \text { and } \epsilon \neq \epsilon^{\prime} \\ 0 & \text { otherwise. }\end{cases}
$$



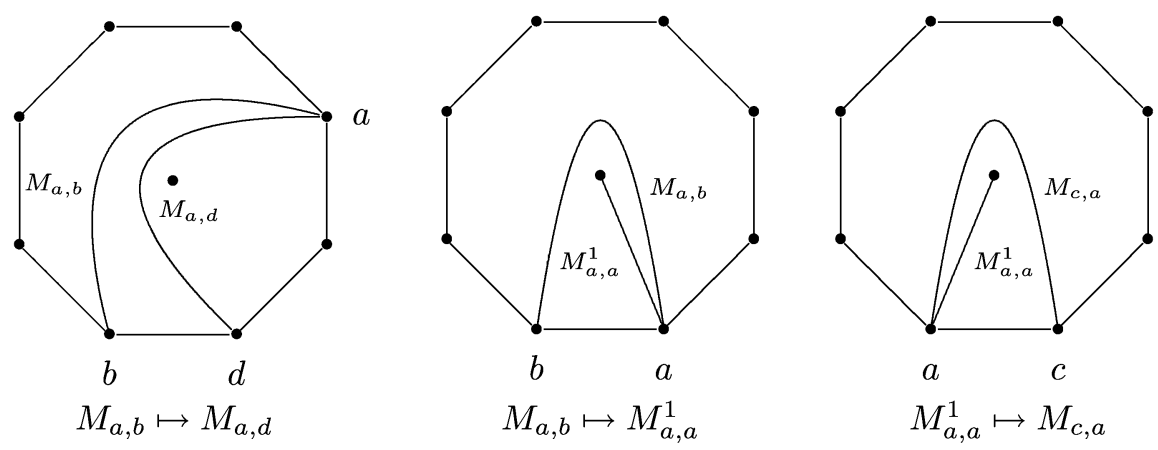

Fig. 2 Examples of elementary moves

Note that $e(M, M)=0$. We say that $M$ crosses $N$ if $e(M, N) \neq 0$. The next lemma immediately follows from the construction.

Lemma 3.3 For any $M, N \in$ ind $\mathcal{C}$, we have $e(M, N) \in\{0,1,2\}$.

\subsection{Elementary moves}

Generalizing a concept from [11], we will now define elementary moves, which will correspond to irreducible morphisms in the cluster category.

An elementary move sends a tagged edge $M_{a, b}^{\epsilon} \in E^{\prime}$ to another tagged edge $M_{a^{\prime}, b^{\prime}}^{\epsilon^{\prime}} \in E^{\prime}$ (i.e., it is an ordered pair $\left(M_{a, b}^{\epsilon}, M_{a^{\prime}, b^{\prime}}^{\epsilon^{\prime}}\right)$ of tagged edges) satisfying certain conditions which we will define in four separate cases according to the relative position of $a$ and $b$. Let $c$ (respectively $d$ ) be the counterclockwise neighbor of $a$ (respectively $b$ ).

1. If $\left|\delta_{a, b}\right|=3$, then there is precisely one elementary move $M_{a, b} \mapsto M_{a, d}$.

2. If $4 \leq\left|\delta_{a, b}\right| \leq n-1$, then there are precisely two elementary moves $M_{a, b} \mapsto M_{c, b}$ and $M_{a, b} \mapsto M_{a, d}$.

3. If $\left|\delta_{a, b}\right|=n$, then $d=a$, and there are precisely three elementary moves $M_{a, b} \mapsto$ $M_{c, b}, M_{a, b} \mapsto M_{a, a}^{1}$, and $M_{a, b} \mapsto M_{a, a}^{-1}$.

4. If $\left|\delta_{a, b}\right|=n+1$, then $a=b$, and there is precisely one elementary move $M_{a, a}^{\epsilon} \mapsto$ $M_{c, a}$.

Some examples of elementary moves are shown in Fig. 2.

3.4 A triangulation of type $D_{n}$

A triangulation of the punctured polygon is a maximal set of noncrossing tagged edges.

Lemma 3.4 Any triangulation of the punctured polygon has $n$ elements.

Proof We prove the lemma by induction on $n$. If $n=3$, there exist, up to rotation, symmetry, and changing tags, three different triangulations (each having 3 tagged edges): 

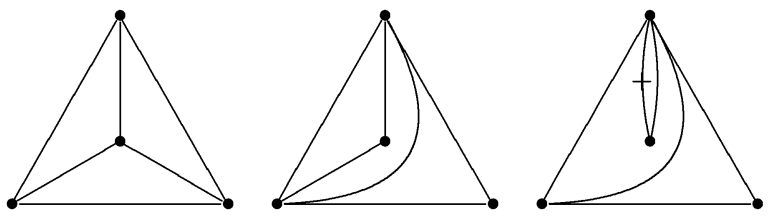

Suppose now that $n>3$, and let $T$ be a triangulation. If $T$ contains an edge of the form $M=M_{a, b}$ with $a \neq b$, then this edge cuts the punctured polygon into two parts. Let $p$ be the number of vertices of the punctured polygon that lie in the part containing the puncture. Then $n-p+2$ vertices lie in the other part, since $a$ and $b$ lie in the both. The set $T \backslash\{M\}$ defines triangulations on both parts. If $p \geq 3$, then, by induction, the number of edges in $T \backslash\{M\}$ that lie in the part containing the puncture is equal to $p$. If $p=2$, then this number is also equal to $p$ (in this case, the 2 edges are either $\left(M_{a, a}^{\epsilon}, M_{a, a}^{-\epsilon}\right),\left(M_{a, a}^{\epsilon}, M_{c, c}^{\epsilon}\right)$, or $\left.\left(M_{c, c}^{\epsilon}, M_{c, c}^{-\epsilon}\right)\right)$. Thus, in all cases, the number of edges in $T \backslash\{M\}$ that lie in the part containing the puncture is equal to $p$. On the other hand, the number of edges in $T \backslash\{M\}$ that lie in other part equals the number of edges in a triangulation of an $(n-p+2)$-polygon, and this number is equal to $n-p-1$. So the cardinality of $T \backslash\{M\}$ is $n-1$, hence $T$ has $n$ elements.

If $T$ does not contain an edge of the form $M_{a, b}$ with $a \neq b$, then $T$ contains exactly one element $M_{a, a}^{\epsilon}$ for each vertex $a$, and all edges have the same tag $\epsilon=1$ or $\epsilon=-1$. Clearly, $T$ has $n$ elements.

We now construct a particular triangulation of the punctured polygon. Let $a_{1}$ be a vertex on the boundary of the punctured polygon. Denote by $a_{2}$ the counterclockwise neighbor of $a_{1}$ and, recursively, let $a_{k}$ be the counterclockwise neighbor of $a_{k-1}$ for any $k$ such that $2 \leq k \leq n$. Then the triangulation $T=T\left(a_{1}\right)$ is the set of tagged edges

$$
T=\left\{M_{a_{1}, a_{1}}^{1}, M_{a_{1}, a_{1}}^{-1}\right\} \cup\left\{M_{a_{1}, a_{k}} \mid 3 \leq k \leq n\right\} .
$$

Here is an example for $T$ in the case $n=8$.

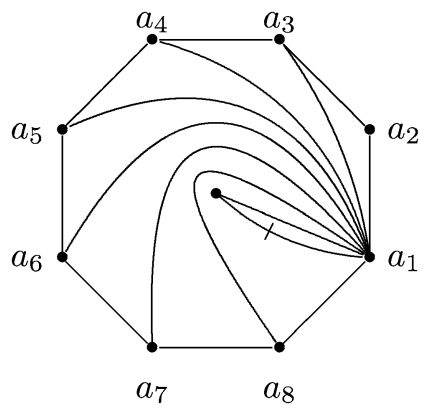

From the construction it is clear that the elementary moves between the elements of $T$ are precisely the elementary moves $M_{a_{1}, a_{k}} \mapsto M_{a_{1}, a_{k+1}}$ for all $k$ with $3 \leq k \leq$ $n-1$ and $M_{a_{1}, a_{n}} \mapsto M_{a_{1}, a_{1}}^{\epsilon}$ with $\epsilon= \pm 1$. We can associate a quiver $Q_{T}$ to $T$ as follows: The vertices of $Q_{T}$ are the elements of $T$, and the arrows are the elementary moves between the elements of $T$. By the above observation, $Q_{T}$ is the following 
quiver of type $D_{n}$ (for $n=3$, set $\left.D_{3}=A_{3}\right)$ :

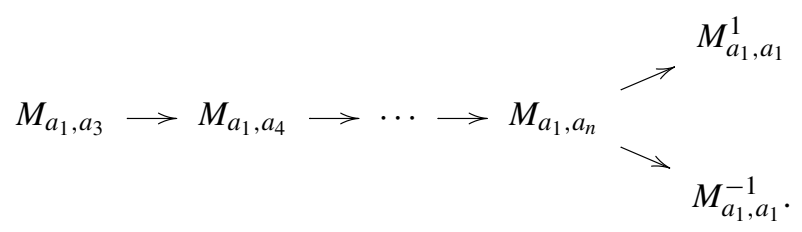

Note that $Q_{T}$ is isomorphic to the quiver $Q$ that we have chosen to construct the cluster category in Sect. 2.3.

\subsection{Translation}

We define the translation $\tau$ to be the following bijection $\tau: E^{\prime} \rightarrow E^{\prime}$ : Let $M_{a, b}^{\epsilon} \in E^{\prime}$, and let $a^{\prime}$ (respectively $b^{\prime}$ ) be the clockwise neighbor of $a$ (respectively $b$ ).

1. If $a \neq b$, then $\tau M_{a, b}=M_{a^{\prime}, b^{\prime}}$.

2. If $a=b$, then $\tau M_{a, a}^{\epsilon}=M_{a^{\prime}, a^{\prime}}^{-\epsilon}$ for $\epsilon= \pm 1$.

The next lemma immediately follows from the definition of $\tau$.

\section{Lemma 3.5}

(1) If $n$ is even, then $\tau^{n}=\mathrm{id}$.

(2) If $n$ is odd, then $\tau^{n} M_{a, b}^{\epsilon}= \begin{cases}M_{a, b}^{\epsilon} & \text { if } a \neq b, \\ M_{a, a}^{-\epsilon} & \text { if } a=b .\end{cases}$

We will need the following lemma when we define the category of tagged edges.

Lemma 3.6 Let $M_{a, b}^{\lambda}, M_{c, d}^{\epsilon} \in E^{\prime}$. Then there is an elementary move $M_{a, b}^{\lambda} \mapsto M_{c, d}^{\epsilon}$ if and only if there is an elementary move $\tau M_{c, d}^{\epsilon} \mapsto M_{a, b}^{\lambda}$.

Proof Let $M_{c^{\prime}, d^{\prime}}^{\epsilon^{\prime}}=\tau M_{c, d}^{\epsilon}$. Suppose that there is an elementary move $M_{a, b}^{\lambda} \mapsto M_{c, d}^{\epsilon}$. Then either $a=c$ or $b=d$.

1. If $a=c$, then $b=d^{\prime}$ and $\left|\delta_{c, d}\right|=\left|\delta_{c^{\prime}, d^{\prime}}\right|=\left|\delta_{a, b}\right|+1$. In particular, $a \neq b$ and $\left|\delta_{c^{\prime}, d^{\prime}}\right| \geq 4$. Now, if $4 \leq\left|\delta_{c^{\prime}, d^{\prime}}\right| \leq n$, then $c^{\prime} \neq d^{\prime}$ and, by 3.3.2, 3.3.3, there is an elementary move $\tau M_{c, d}=M_{c^{\prime}, b} \mapsto M_{c, b}=M_{a, b}$. On the other hand, if $\left|\delta_{c^{\prime}, d^{\prime}}\right|=n+1$, then $c^{\prime}=d^{\prime}=b$ and $a$ is the counterclockwise neighbor of $b$, and thus, by 3.3.4, there is an elementary move $\tau M_{c, d}^{\epsilon}=M_{b, b}^{-\epsilon} \mapsto M_{a, b}$.

2. If $b=d$, then $a=c^{\prime}$ and $\left|\delta_{c, d}\right|=\left|\delta_{c^{\prime}, d^{\prime}}\right|=\left|\delta_{a, b}\right|-1$. In particular, $\left|\delta_{c^{\prime}, d^{\prime}}\right| \leq n$. Now, if $3 \leq\left|\delta_{c^{\prime}, d^{\prime}}\right| \leq n-1$, then, by $3.3 .1,3.3 .2$, there is an elementary move $\tau M_{c, d}=M_{a, d^{\prime}} \mapsto M_{a, b}$. On the other hand, if $\left|\delta_{c^{\prime}, d^{\prime}}\right|=n$, then $a=b=d$ and $b$ is the counterclockwise neighbor of $d^{\prime}$, and thus, by 3.3.3, there is an elementary move $\tau M_{c, d}^{\epsilon}=M_{a, d^{\prime}} \mapsto M_{a, b}^{\lambda}$ for $\lambda= \pm 1$.

Thus, there is an elementary move $\tau M_{c, d}^{\epsilon} \mapsto M_{a, b}^{\lambda}$. By symmetry, the other implication also holds. 


\subsection{The category $\mathcal{C}$}

We will now define a $k$-linear additive category $\mathcal{C}$ as follows. The objects are direct sums of tagged edges in $E^{\prime}$. By additivity, it suffices to define morphisms between tagged edges. The space of morphisms from a tagged edge $M$ to a tagged edge $N$ is a quotient of the vector space over $k$ spanned by sequences of elementary moves starting at $M$ and ending at $N$. The subspace which defines the quotient is spanned by the so-called mesh relations.

For any tagged edge $X \in E^{\prime}$, we define the mesh relation

$$
m_{X}=\sum_{\alpha}(\alpha) \alpha,
$$

where the sum is over all elementary moves $Y \stackrel{\alpha}{\mapsto} X$ that send a tagged edge $Y$ to $X$, and $\tau X \stackrel{(\alpha)}{\mapsto} Y$ denotes the elementary move given by Lemma 3.6. In other terms, if there is only one $Y \in E^{\prime}$ such that there is an elementary move $Y \stackrel{\alpha}{\mapsto} X$, then the composition of morphisms $\tau X \stackrel{(\alpha)}{\mapsto} Y \stackrel{\alpha}{\mapsto} X$ is zero, and if there are several $Y_{1}, \ldots, Y_{p} \in E^{\prime}$

with elementary moves $Y_{i} \stackrel{\alpha_{i}}{\mapsto} X$, then the sum of all compositions of morphisms $\tau X \stackrel{\left(\alpha_{i}\right)}{\mapsto} Y_{i} \stackrel{\alpha_{i}}{\mapsto} X, i=1, \ldots, n$, is zero.

More generally, a mesh relation is an equality between sequences of elementary moves which differ precisely by such a relation $m_{X}$.

We can now define the set of morphisms from a tagged edge $M$ to a tagged edge $N$ to be the quotient of the vector space over $k$ spanned by sequences of elementary moves from $M$ to $N$ by the subspace generated by mesh relations.

\section{Equivalence of categories}

In this section, we will prove the equivalence of the category $\mathcal{C}$ and the cluster category $\mathcal{C}_{A}$.

\subsection{Translation quiver}

First we construct a stable translation quiver in our situation. Let $\Gamma=\left(\Gamma_{0}, \Gamma_{1}\right)$ be the following quiver: The set of vertices is the set $\mathbb{Z} \times E^{\prime}$, where $E^{\prime}$ is the set of tagged edges of Sect. 3.1. In order to define the set of arrows, let us fix a vertex $a_{1}$ on the boundary of the punctured polygon, and let $T=T\left(a_{1}\right)$ be the triangulation of Sect. 3.4. Given $M, N \in E^{\prime}$, there is an arrow $(i, M) \rightarrow(i, N)$ in $\Gamma_{1}$ if there is an elementary move $M \mapsto N$ and either $N \notin T$ or $M$ and $N$ are both in $T$, and there is an arrow $(i, M) \rightarrow(i+1, N)$ in $\Gamma_{1}$ if there is an elementary move $M \mapsto N$ and $N \in T$ and $M \notin T$. Note that $\Gamma$ has no loops and no multiple arrows.

The translation $\tau: E^{\prime} \rightarrow E^{\prime}$ defined in Sect. 3.5 induces a bijection $\tau_{\Gamma}$ on $\Gamma_{0}$ by

$$
\tau_{\Gamma}(i, M)= \begin{cases}(i, \tau M) & \text { if } M \notin T, \\ (i-1, \tau M) & \text { if } M \in T .\end{cases}
$$


Then from Lemma 3.6 it follows that $\left(\Gamma, \tau_{\Gamma}\right)$ is a stable translation quiver. Let $\mathcal{M}\left(\Gamma, \tau_{\Gamma}\right)$ denote the mesh category of $\left(\Gamma, \tau_{\Gamma}\right)$.

Now consider the vertex subset $0 \times T \subset \Gamma_{0}$. This set contains exactly one element of each $\tau_{\Gamma}$-orbit of $\Gamma$. Moreover, the full subquiver induced by $0 \times T$ is the quiver $Q=Q_{T}$ of Sects. 2.3 and 3.4, a quiver of type $D_{n}$, and from Sect. 3.3 it follows that $\Gamma$ is the translation quiver $\mathbb{Z} Q$. Let $A$ be the path algebra of $Q$, as before, and let us use the notation ( $)^{-}$of Sect. 2.3. Then, by Proposition 2.1, we have the following result.

Proposition 4.1 There is an equivalence of categories

$$
\varphi: \mathcal{M}\left(\Gamma, \tau_{\Gamma}\right) \stackrel{\sim}{\longrightarrow} \text { ind } \mathcal{D}^{b} A
$$

such that

1. $\varphi$ maps the elements of $0 \times T$ to the projective A-modules.

2. $\varphi \circ \tau_{\Gamma}=\tau_{\mathcal{D}^{b} A} \circ \varphi$.

3. If $\operatorname{level}\left(\varphi\left(\ell, M_{a, b}^{\epsilon}\right)\right)=j$, then

$$
j=\left|\delta_{a, b}\right|-2 \quad \text { if } a \neq b \quad \text { and } \quad j \in\{n-1, n\} \quad \text { if } a=b .
$$

4. If $M=\left(\ell, M_{a, a}^{\epsilon}\right)$ and $M^{-}=\left(\ell, M_{a, a}^{-\epsilon}\right)$, then

$$
(\varphi(M))^{-}=\varphi\left(M^{-}\right)
$$

The equivalence $\varphi$ induces an equivalence of categories $\varphi^{\prime}: \oplus \mathcal{M}\left(\Gamma, \tau_{\Gamma}\right) \stackrel{\sim}{\rightarrow} \mathcal{D}^{b} A$, where $\oplus \mathcal{M}\left(\Gamma, \tau_{\Gamma}\right)$ denotes the additive hull of $\mathcal{M}\left(\Gamma, \tau_{\Gamma}\right)$. In particular, $\oplus \mathcal{M}\left(\Gamma, \tau_{\Gamma}\right)$ has a triangulated structure such that $\varphi^{\prime}$ is an equivalence of triangulated categories. Let $\rho$ be the endofunctor of $\oplus \mathcal{M}\left(\Gamma, \tau_{\Gamma}\right)$ given by the full counterclockwise rotation of the punctured polygon. Thus, if $(i, M) \in \mathcal{M}\left(\Gamma, \tau_{\Gamma}\right)$, then $\rho(i, M)=(i+1, M)$. The next result follows from Lemma 3.5.

Lemma 4.2 (1) If $n$ is even, then $\rho=\tau_{\Gamma}^{-n}$.

(2) If $n$ is odd and $M=\left(\ell, M_{a, b}\right)$ with $a \neq b$, then $\rho(M)=\tau_{\Gamma}^{-n}(M)$.

(3) If $n$ is odd and $M=\left(\ell, M_{a, a}^{\epsilon}\right)$ and $M^{-}=\left(\ell, M_{a, a}^{-\epsilon}\right)$, then $\rho(M)=\tau_{\Gamma}^{-n}\left(M^{-}\right)$.

\subsection{Main result}

The category of tagged edges $\mathcal{C}$ of Sect. 3.6 is the quotient category of the triangulated category $\oplus \mathcal{M}\left(\Gamma, \tau_{\Gamma}\right)$ by the endofunctor $\rho$. Hence the objects of $\mathcal{C}$ are the $\rho$-orbits $\tilde{M}=\left(\rho^{i} M\right)_{i \in \mathbb{Z}}$ of objects $M$ in $\oplus \mathcal{M}\left(\Gamma, \tau_{\Gamma}\right)$, and $\operatorname{Hom}_{\mathcal{C}}(\tilde{M}, \tilde{N})=$ $\oplus_{i} \operatorname{Hom}\left(M, \rho^{i} M\right)$, where the Hom-spaces on the right are taken in the category $\oplus \mathcal{M}\left(\Gamma, \tau_{\Gamma}\right)$. We are now able to show our main result.

Theorem 4.3 There is an equivalence of categories

$$
\bar{\varphi}: \mathcal{C} \longrightarrow \mathcal{C}_{A}
$$

between the category of tagged edges $\mathcal{C}$ and the cluster category $\mathcal{C}_{A}$ which sends the $\rho$-orbit of the triangulation $0 \times T$ to the $F$-orbit of the projective A-modules. 
Proof Let $\varphi^{\prime}: \oplus \mathcal{M}\left(\Gamma, \tau_{\Gamma}\right) \rightarrow \mathcal{D}^{b} A$ be the equivalence of Sect. 4.1. Since $C=$ $\oplus \mathcal{M}\left(\Gamma, \tau_{\Gamma}\right) / \rho$ and $\mathcal{C}_{A}=\mathcal{D}^{b} A / F$, we only have to show that $\varphi(\rho(M))=F(\varphi(M))$ for any $M \in \mathcal{M}\left(\Gamma, \tau_{\Gamma}\right)$.

If $n$ is even, then $F \varphi=\tau_{\mathcal{D}^{b} A}^{-1}[1] \varphi=\tau_{\mathcal{D}^{b} A}^{-n} \varphi$ by Lemma 2.2. On the other hand, $\varphi \rho=\varphi \tau_{\Gamma}^{-n}$ by Lemma 4.2, and then the statement follows from Proposition 4.1.

Suppose now that $n$ is odd. Let $M=\left(\ell, M_{a, b}^{\epsilon}\right) \in \mathcal{M}\left(\Gamma, \tau_{\Gamma}\right)$. If $a \neq b$, then level $(\varphi(M))=\left|\delta_{a, b}\right|-2 \leq n-2$ by Proposition 4.1, and, therefore, $F(\varphi(M))=$ $\tau_{\mathcal{D}^{b} A}^{-n} \varphi(M)$ by Lemma 2.2. On the other hand, Lemma 4.2 implies that $\varphi \rho(M)=$ $\varphi \tau_{\Gamma}^{-n}(M)$, and the statement follows from Proposition 4.1. Finally, suppose that $a=b$, that is, $M=\left(\ell, M_{a, a}^{\epsilon}\right)$ and $\operatorname{level}(\varphi(M)) \in\{n-1, n\}$. Let $M^{-}=\left(\ell, M_{a, a}^{-\epsilon}\right)$; then, by Proposition 4.1, we have $(\varphi(M))^{-}=\varphi\left(M^{-}\right)$and thus $F(\varphi(M))=$ $\tau_{\mathcal{D}^{b} A}^{-n} \varphi\left(M^{-}\right)$by Lemma 2.2. On the other hand, Lemma 4.2 implies that $\varphi \rho(M)=$ $\varphi \tau_{\Gamma}^{-n}\left(M^{-}\right)$, and again the statement follows from Proposition 4.1.

Remark 4.4 It has been shown in [21] that the cluster category $\mathcal{C}_{A}$ is triangulated. The shift functor [1] of this triangulated structure is induced by the shift functor of $\mathcal{D}^{b} A$ and [1] $=\tau_{\mathcal{C}_{A}}$, by construction.

Thus the category of tagged edges is also triangulated and its shift functor is equal to $\tau$. In particular, we can define the $\operatorname{Ext}^{1}$ of two objects $M, N \in \operatorname{ind} \mathcal{C}$ as

$$
\operatorname{Ext}_{\mathcal{C}}^{1}(M, N)=\operatorname{Hom}_{\mathcal{C}}(M, \tau N) .
$$

We study Ext ${ }^{1}$ in the next section.

\section{Dimension of $\mathbf{E x t}^{1}$}

We want to translate the statement of Proposition 2.3 in the category $\mathcal{C}$. For an element $N \in \operatorname{ind} \mathcal{C}$, let $\operatorname{pos}(N)=(i, j)$ be the position of the corresponding indecomposable object $\bar{\varphi}(N)$ in $\mathcal{C}_{A}$ under the equivalence $\bar{\varphi}$ of Theorem 4.3. For two vertices $a, b$ on the boundary of the punctured polygon, define the closed interval $[a, b]$ to be the set of all vertices that lie on the counterclockwise path from $a$ to $b$ on the boundary. The open interval $] a, b[$ is $[a, b] \backslash\{a, b\}$. Recall that $e(M, N)$ denotes the crossing number of tagged edges $M, N$ (see Sect. 3.2). The various cases of the following lemma are illustrated in Figs. 3 and 4.

Lemma 5.1 Let $M_{a, b} \in$ ind $\mathcal{C}$ with $a \neq b$ be such that $\operatorname{pos}\left(M_{a, b}\right)=(1, m)$. Let $M_{x, y}^{\epsilon} \in \operatorname{ind} \mathcal{C}$ be arbitrary, and denote $\operatorname{pos}\left(M_{x, y}^{\epsilon}\right)$ by $(i, j)$. Then

1. $e\left(\tau M_{a, b}, M_{x, y}^{\epsilon}\right) \neq 0$ if and only if one of the following conditions hold:

$$
\begin{array}{ccc}
1 \leq i \leq m & \& & m+1 \leq i+j \\
m+1 \leq i \leq n-1 & \& & n \leq i+j \leq n+m-1 .
\end{array}
$$

2. $e\left(\tau M_{a, b}, M_{x, y}^{\epsilon}\right)=2$ if and only if

$$
m \geq 2 \quad \& \quad 2 \leq i \leq m \quad \& \quad n \leq i+j \quad \& \quad 2 \leq j \leq n-2 .
$$




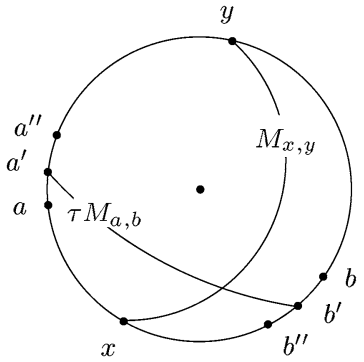

$x \in\left[a, b^{\prime \prime}\right]$

$y \in[b, x]$

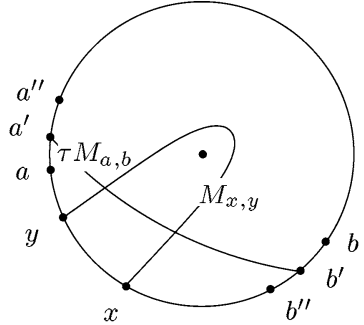

$$
\begin{aligned}
& x \in\left[a, b^{\prime \prime}\right] \\
& y \in[b, x]
\end{aligned}
$$

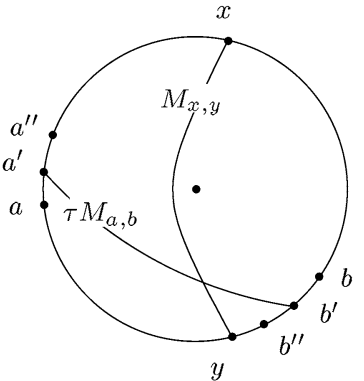

$x \in\left[b, a^{\prime \prime}\right]$ $y \in\left[a, b^{\prime \prime}\right]$

Fig. 3 Proof of Lemma 5.1.1

Fig. 4 Lemma 5.1.2

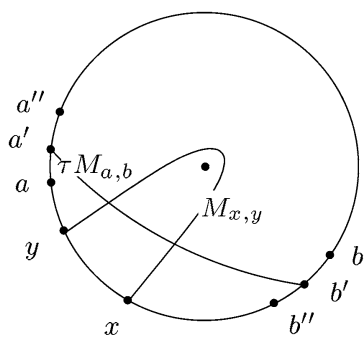

$$
\begin{aligned}
& x \in] a, b^{\prime}[ \\
& y \in] a^{\prime}, x[
\end{aligned}
$$

Proof Let $M_{a, b}$ be as in the lemma. By Proposition 4.1, we have $1 \leq m=\left|\delta_{a, b}\right|-2 \leq$ $n-2$. We use the notation $\tau M_{a, b}=M_{a^{\prime}, b^{\prime}}$ and $\tau^{2} M_{a, b}=M_{a^{\prime \prime}, b^{\prime \prime}}$. Let $M_{x, y}^{\epsilon} \in$ ind $\bar{C}$ be such that $\operatorname{pos}\left(M_{x, y}^{\epsilon}\right)=(i, j)$. Then $e\left(\tau M_{a, b}, M_{x, y}^{\epsilon}\right) \neq 0$ if and only if one of the following conditions hold (see Fig. 3):

$$
\begin{array}{cll}
x \in\left[a, b^{\prime \prime}\right] & \& & y \in[b, x], \\
x \in\left[b^{\prime}, a^{\prime \prime}\right] & \& & y \in\left[a, b^{\prime \prime}\right] .
\end{array}
$$

In Fig. 3, the two drawings on the left both correspond to the case (1) and the drawing on the right to the case (2). We will now rewrite these conditions in terms of $i$ and $j$. First note that $i=\left|\delta_{a, x}\right|$. Moreover, if $x \neq y$, then Proposition 4.1 implies that $j=$ $\left|\delta_{x, y}\right|-2$; and if $x=y$, then $n-1 \leq j \leq n$. Thus conditions (1) and (2) become

$$
\begin{aligned}
& 1 \leq i \leq\left|\delta_{a, b^{\prime \prime}}\right| \quad \& \quad\left|\delta_{a, b}\right|-1 \leq i+j \leq\left|\delta_{a, x}\right|+n, \\
& \left|\delta_{a, b^{\prime}}\right| \leq i \leq\left|\delta_{a, a^{\prime \prime}}\right| \quad \& \quad\left|\delta_{a, a}\right|-1 \leq i+j \leq\left|\delta_{a, a^{\prime \prime}}\right|+\left|\delta_{a^{\prime \prime}, b^{\prime \prime}}\right|-2,
\end{aligned}
$$

where $(1) \Leftrightarrow(3)$ and $(2) \Leftrightarrow(4)$. Note that the upper bound for $i+j$ in (3) is always satisfied, since $j \leq n$ and $i=\left|\delta_{a, x}\right|$. Now the first statement of the lemma follows simply by counting the vertices on the various boundary paths that appear in (3) and (4). 


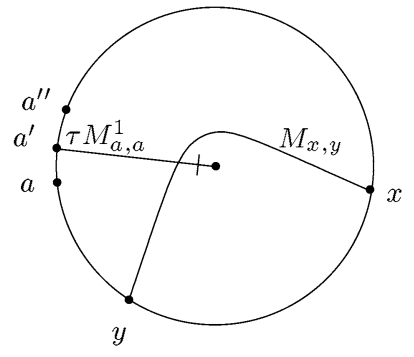

$$
\begin{aligned}
& \left.x \in] a, a^{\prime \prime}\right] \\
& y \in[a, x[
\end{aligned}
$$

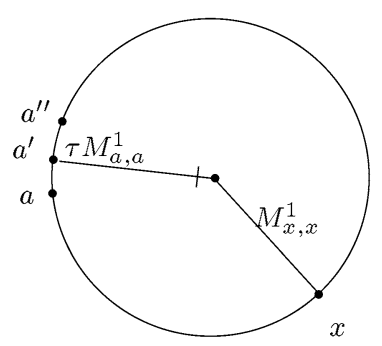

$$
\begin{aligned}
& x=y \neq a^{\prime} \\
& \epsilon=\epsilon^{\prime}
\end{aligned}
$$

Fig. 5 Two cases of Lemma 5.2

On the other hand, $e\left(\tau M_{a, b}, M_{x, y}^{\epsilon}\right)=2$ if and only if

$$
x \in] a, b^{\prime}[\quad \& \quad y \in] a^{\prime}, x[
$$

(see Fig. 4). Note that $x$ cannot be equal to $a$, since otherwise $a=x=y$ and then, by the definition of the crossing number, $e\left(\tau M_{a, b}^{\epsilon}, M_{x, y}^{\epsilon}\right) \leq 1$. In particular, we have $m=\left|\delta_{a^{\prime}, b^{\prime}}\right|-2 \geq 2$. Again, using the fact that $i=\left|\delta_{a, x}\right|$ and $j=\left|\delta_{x, y}\right|-2$, we see that $e\left(\tau M_{a, b}, M_{x, y}^{\epsilon}\right)=2$ if and only if

$$
m \geq 2 \quad \& \quad 2 \leq i \leq\left|\delta_{a, b^{\prime}}\right|-1 \quad \& \quad\left|\delta_{a, a}\right|-1 \leq i+j \quad \& \quad 2 \leq j \leq n-2 .
$$

Now, the second statement of the lemma follows, since $\left|\delta_{a, b^{\prime}}\right|-1=m$.

The two cases of the following lemma are illustrated in Fig. 5.

Lemma 5.2 Let $M_{a, a}^{\epsilon} \in \operatorname{ind} \mathcal{C}$ be such that $\operatorname{pos}\left(M_{a, a}^{\epsilon}\right)=(1, m)$ with $m \in\{n-1, n\}$. Define $m^{\prime}$ to be the unique element in $\{n-1, n\} \backslash\{m\}$. Let $M_{x, y}^{\epsilon^{\prime}} \in$ ind $\mathcal{C}$ be arbitrary, and denote $\operatorname{pos}\left(M_{x, y}^{\epsilon^{\prime}}\right)$ by $(i, j)$. Then

1. $e\left(\tau M_{a, a}^{\epsilon}, M_{x, y}^{\epsilon^{\prime}}\right)=1$ if and only if one of the following conditions hold:

$$
\begin{aligned}
& 2 \leq i \leq n-1 \quad \& \quad i+j \geq n \quad \& \quad j \leq n-2, \\
& 1 \leq i \leq n-1 \quad \& \quad j= \begin{cases}m^{\prime} & \text { if } i \text { is odd }, \\
m & \text { if } i \text { is even } .\end{cases}
\end{aligned}
$$

2. $e\left(\tau M_{a, a}^{\epsilon}, M_{x, y}^{\epsilon^{\prime}}\right)=0$ otherwise.

Proof Let $M_{a, a}^{\epsilon}$ be as in the lemma, and let $\tau M_{a, a}^{\epsilon}=M_{a^{\prime}, a^{\prime}}^{-\epsilon}$ and $\tau^{2} M_{a, a}^{\epsilon}=M_{a^{\prime \prime}, a^{\prime \prime}}^{\epsilon}$. Let $M_{x, y}^{\epsilon^{\prime}} \in \operatorname{ind} \mathcal{C}$ be such that $\operatorname{pos}\left(M_{x, y}^{\epsilon^{\prime}}\right)=(i, j)$. By the definition of the crossing number we have that $e\left(\tau M_{a, a}^{\epsilon}, M_{x, y}^{\epsilon^{\prime}}\right)=1$ if and only if one of the following conditions hold (see Fig. 5): 


$$
\begin{array}{lll}
\left.x \in] a, a^{\prime \prime}\right] & \& & y \in[a, x[, \\
x=y \neq a^{\prime} & \& & \epsilon=\epsilon^{\prime} .
\end{array}
$$

We can rewrite these conditions in terms of $i$ and $j$ as follows:

$$
\begin{aligned}
& 2 \leq i \leq\left|\delta_{a, a^{\prime \prime}}\right| \quad \& \quad\left|\delta_{a, a}\right|-1 \leq i+j \quad \& \quad j \leq\left|\delta_{x, x}\right|-3, \\
& 1 \leq i \leq n-1 \quad \& \quad j= \begin{cases}m & \text { if } i \text { is odd, } \\
m^{\prime} & \text { if } i \text { is even. }\end{cases}
\end{aligned}
$$

Counting the vertices on the boundary paths yields the lemma.

Theorem 5.3 Let $M, N \in \operatorname{ind} \mathcal{C}$. Then the dimension of $\operatorname{Ext}_{\mathcal{C}}^{1}(M, N)$ is equal to the crossing number $e(M, N)$ of $M$ and $N$.

Remark 5.4 Note that the analogue of Theorem 5.3 also holds in type $A_{n}$; see [11].

Proof We can assume without loss of generality that $\operatorname{pos}(M)=(1, m)$ for some $m \in\{1, \ldots, n\}$. Let $(i, j)$ denote $\operatorname{pos}(N)$. Because of the equivalence of categories of Theorem 4.3, we can calculate the dimension of $\operatorname{Hom}_{\mathcal{C}}(M, N)$ in terms of the positions $\operatorname{pos}(M), \operatorname{pos}(N)$, using the formulas in Proposition 2.3. Comparing this result with the description of $e(\tau M, N)$ in Lemmas 3.3, 5.1, and 5.2, we conclude that the dimension of $\operatorname{Hom}_{\mathcal{C}}(M, N)$ is equal to $e(\tau M, N)$ for all $M, N \in \operatorname{ind} \mathcal{C}$. Hence $e(M, N)=\operatorname{dim}_{H_{C}}\left(\tau^{-1} M, N\right)=\operatorname{dim}_{H_{C}}(M, \tau N)$. On the other hand, $\operatorname{Ext}_{\mathcal{C}}^{1}(M, N)=\operatorname{Hom}_{\mathcal{C}}(M, \tau N)$ by definition, and the result follows.

Remark 5.5 Since the crossing number is symmetric, this theorem illustrates the symmetry of $\mathrm{Ext}^{1}$ in the cluster category, that is, for any $M, N \in \operatorname{ind} \mathcal{C}$, we have

$$
\operatorname{dim} \operatorname{Ext}_{\mathcal{C}}^{1}(M, N)=\operatorname{dim}_{\operatorname{Ext}_{\mathcal{C}}^{1}}^{1}(N, M) .
$$

\section{Applications}

\subsection{Auslander-Reiten-triangles}

By [21] and Theorem 4.3, $\mathcal{C}$ is a triangulated category with Auslander-Reitentriangles

$$
\tau M \rightarrow L \rightarrow M \rightarrow \tau M[1]=\tau^{2} M
$$

We have described $\tau$ already; let us now describe $L$.

1. Suppose that $\tau M=M_{a, b}$ with $a \neq b$. Then $M=M_{c, d}$, where $c$ (respectively $d$ ) is the counterclockwise neighbor of $a$ (respectively $b$ ). In particular, $c \neq b$, since $\left|\delta_{a, b}\right| \geq 3$.

If $a \neq d$, then $L$ is the direct sum of $M_{c, b}$ and $M_{a, d}$, and if $a=d$, then $L$ is the direct sum of $M_{c, b}, M_{a, a}^{1}$ and $M_{a, a}^{-1}$. 


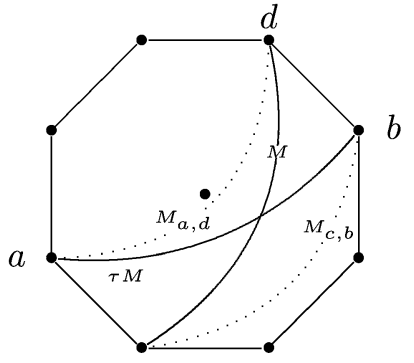

c

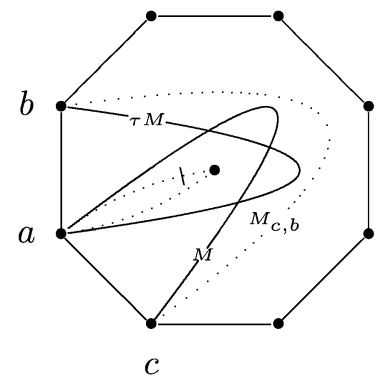

$a=d$

$a \neq d$

2. Suppose that $\tau M=M_{a, a}^{-\epsilon}$. Then $M=M_{c, c}^{\epsilon}$, where $c$ is the counterclockwise neighbor of $a$. Then $L$ is indecomposable, $L=M_{c, a}$.

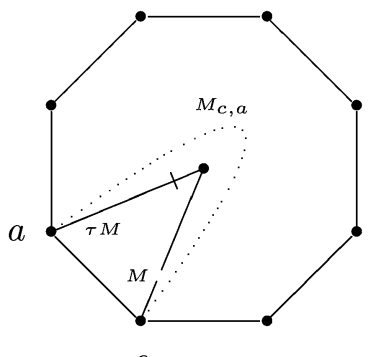

c

Note that there are irreducible morphisms $\tau M \rightarrow L$ and $L \rightarrow M$, and $\tau M \rightarrow L \rightarrow M$ is a mesh.

Remark 6.1 Let us point out that, in type $A_{n}$, all Auslander-Reiten triangles are of type 1 with $a \neq b$; see [11].

\subsection{Tilting objects and exchange pairs}

A tilting object $T$ in the category $\mathcal{C}$ is a maximal set of noncrossing tagged edges, that is, $T$ is a triangulation of the punctured polygon. Let $M, N \in E^{\prime}$. If $e(M, N)=1$, then, by [6, Theorem 7.5], $M, N$ form an exchange pair, that is, there exist two triangulations $T$ and $T^{\prime}$ such that $M \in T, N \in T^{\prime}$, and $T \backslash\{M\}=T^{\prime} \backslash\{N\}$. The edges $M$ and $N$ are the "diagonals" in a generalized quadrilateral in $T \backslash\{M\}$; see Fig. 6. The triangulation $T^{\prime}$ is obtained from the triangulation $T$ by "flipping" the diagonal $M$ to the diagonal $N$.

Let $x_{M}, x_{N}$ be the corresponding cluster variables in the cluster algebra; then the exchange relation is given by

$$
x_{M} x_{N}=\prod_{i} x_{L_{i}}+\prod_{i} x_{L_{i}^{\prime}}
$$



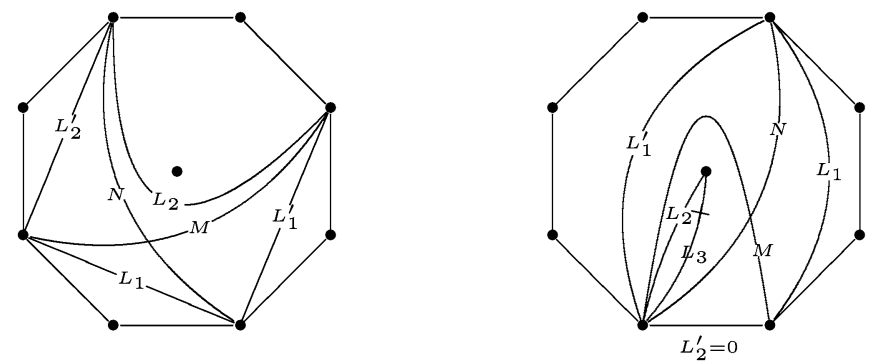

Fig. 6 Two examples of exchange pairs
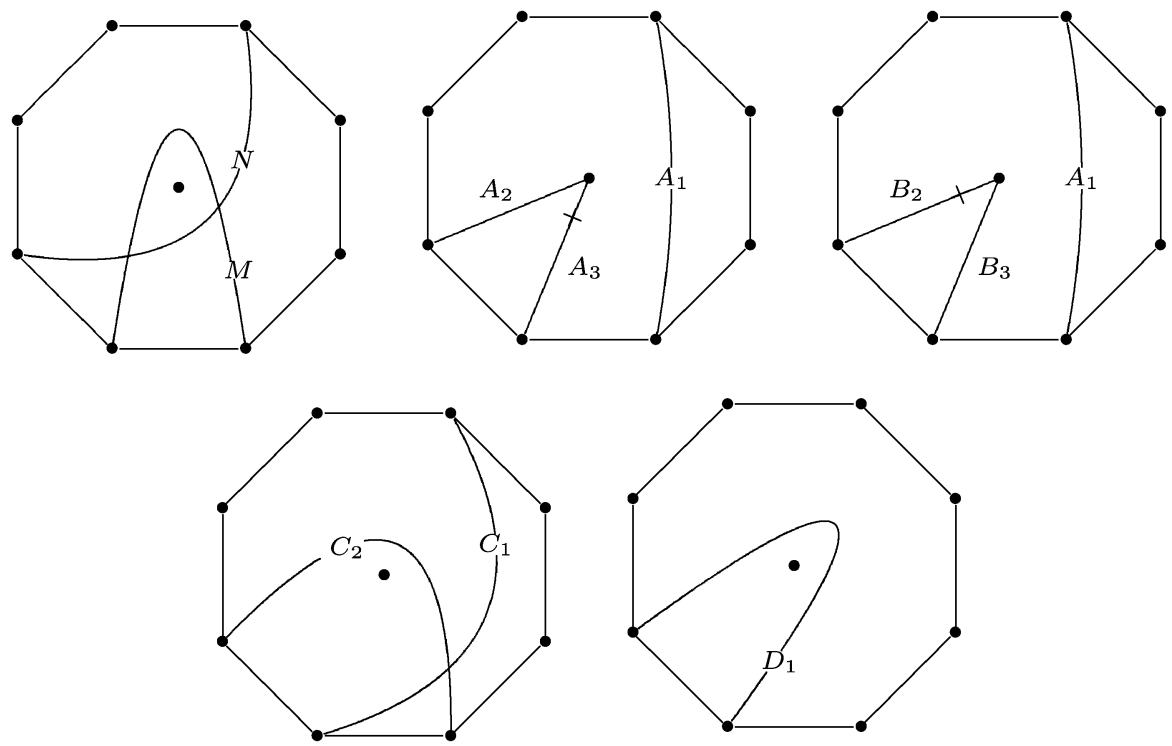

Fig. $7 \mathrm{Ext}^{1}$ of dimension 2

where the products correspond to "opposite" sides in the generalized quadrilateral and can have one, two, or three factors; see Fig. 6.

\section{3 $\mathrm{Ext}^{1}$ of higher dimension}

We include an example of two indecomposables $M, N \in$ ind $\mathcal{C}$ for which the dimension of $\operatorname{Ext}_{\mathcal{C}}^{1}(M, N)$ is equal to two; see Fig. 7. In the same figure, we illustrate indecomposable objects $A_{1}, A_{2}, A_{3}, B_{2}, B_{3}, C_{1}, C_{2}$, and $D_{1}$ that appear in the following triangles:

$$
\begin{array}{cc}
N \rightarrow A_{1} \oplus A_{2} \oplus A_{3} \rightarrow M \rightarrow N[1], \quad N \rightarrow A_{1} \oplus B_{2} \oplus B_{3} \rightarrow M \rightarrow N[1] \\
N \rightarrow C_{1} \oplus C_{2} \rightarrow M \rightarrow N[1], & N \rightarrow D_{1} \rightarrow M \rightarrow N[1] .
\end{array}
$$




\subsection{Cluster-tilted algebra}

Let $T$ be any triangulation of the punctured polygon. Then the endomorphism al-

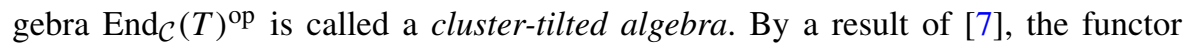
$\operatorname{Hom}_{\mathcal{C}}\left(\tau^{-1} T,-\right)$ induces an equivalence of categories $\varphi_{T}: \mathcal{C} / T \rightarrow \bmod _{\operatorname{End}_{\mathcal{C}}}(T)^{\mathrm{op}}$. Labeling the edges in $T$ by $T_{1}, T_{2}, \ldots, T_{n}$, we get the dimension vector $\underline{\operatorname{dim}} \varphi_{T}\left(M_{a, b}^{\epsilon}\right)$ of a module $\varphi_{T}\left(M_{a, b}^{\epsilon}\right)$ by

$$
\begin{aligned}
\left(\underline{\operatorname{dim}} \varphi_{T}\left(M_{a, b}^{\epsilon}\right)\right)_{i} & =\operatorname{dim} \operatorname{Hom}_{\mathcal{C}}\left(\tau^{-1} T_{i}, M_{a, b}^{\epsilon}\right) \\
& =\operatorname{dim}_{E^{\prime}}^{1}\left(M_{a, b}^{\epsilon}, T_{i}\right)=e\left(M_{a, b}^{\epsilon}, T_{i}\right),
\end{aligned}
$$

where the last equation holds by Theorem 5.3.

We illustrate this in an example. Let $n=4$, and let $T$ be the triangulation

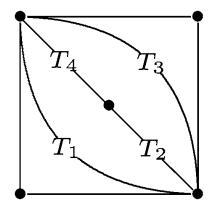

Then the cluster-tilted algebra $\operatorname{End}_{\mathcal{C}}(T)^{\text {op }}$ is the quotient of the path algebra of the quiver

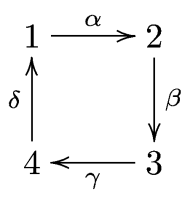

by the ideal generated by the paths $\alpha \beta \gamma, \beta \gamma \delta, \gamma \delta \alpha$, and $\delta \alpha \beta$. The AuslanderReiten quiver of the category $\mathcal{C}$ is

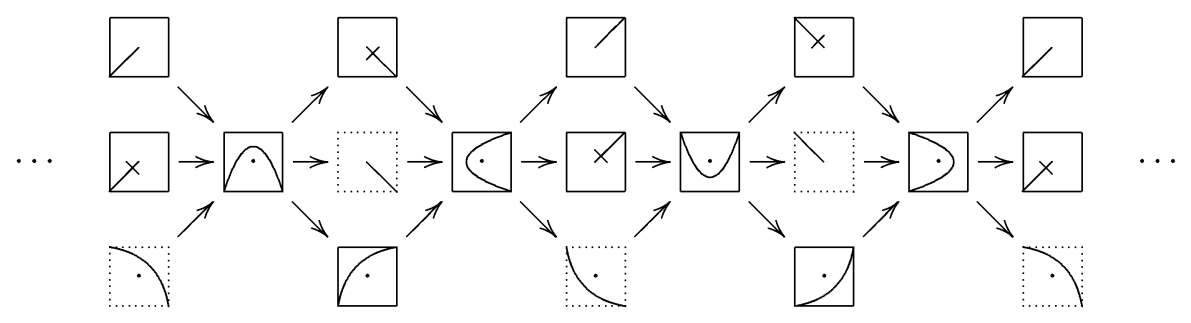

For the four tagged edges of the triangulation $T$, we have drawn the borders of the punctured polygons in the above picture as dotted lines. Deleting these positions and 
using (5), we obtain the Auslander-Reiten quiver of $\operatorname{End}_{\mathcal{C}}(T)^{\text {op }}$

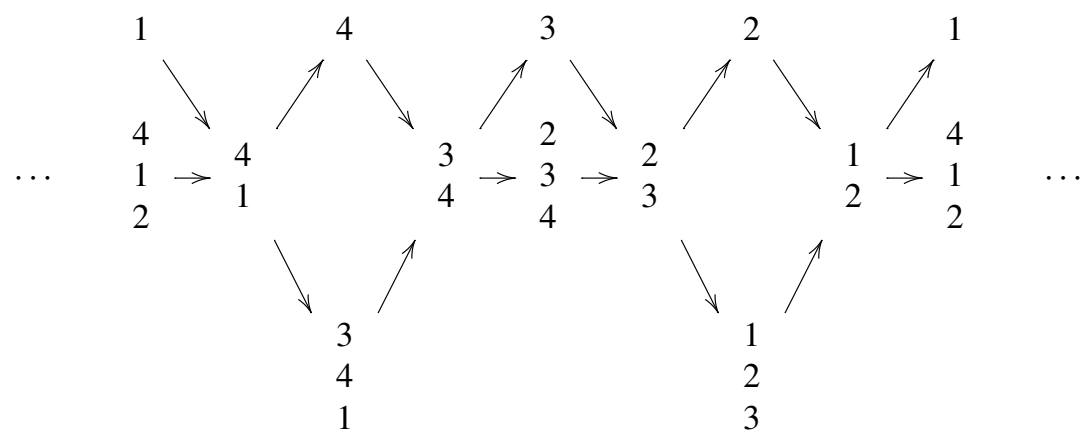

where modules are represented by their Loewy series.

Acknowledgements The author thanks Philippe Caldero, Frederic Chapoton, and Dylan Thurston for interesting discussions on the subject.

\section{References}

1. Assem, I., Brüstle, T., Schiffler, R., \& Todorov, G. (2006). Cluster categories and duplicated algebras. J. Algebra, 305, 547-561. arXiv:math.RT/0509501.

2. Assem, I., Brüstle, T., Schiffler, R., \& Todorov, G. (2006). m-cluster categories and m-replicated algebras. Preprint. arXiv:math.RT/0608727.

3. Assem, I., Simson, D., \& Skowronski, A. (2006). Elements of the representation theory of associative algebras, 1: techniques of representation theory. London mathematical society student texts, Vol. 65. Cambridge: Cambridge University Press.

4. Auslander, M., Reiten, I., \& Smalø, S. O. (1995). Representation theory of Artin algebras. Cambridge studies in advanced math., Vol. 36. Cambridge: Cambridge University Press.

5. Baur, K., \& Marsh, R. (2006). A geometric description of $m$-cluster categories. Trans. Am. Math. Soc., to appear. arXiv:math.RT/0607151.

6. Buan, A., Marsh, R., Reineke, M., Reiten, I., \& Todorov, G. (2006). Tilting theory and cluster combinatorics. Adv. Math., 204, 572-612. arXiv:math.RT/0402054.

7. Buan, A. B., Marsh, R., \& Reiten, I. (2007). Cluster-tilted algebras. Trans. Am. Math. Soc., 359, 323-332. arXiv:math.RT/0402075.

8. Buan, A. B., Marsh, R., \& Reiten, I. Cluster mutation via quiver representations. Comment. Math. Helv., to appear. arXiv:math.RT/0412077.

9. Buan, A., Marsh, R., Reiten, I., \& Todorov, G. Clusters and seeds in acyclic cluster algebras. Proc. Am. Math. Soc., to appear. arXiv:math.RT/0510359.

10. Caldero, P., \& Chapoton, F. (2006). Cluster algebras as Hall algebras of quiver representations. Comment. Math. Helv., 81(3), 595-616. arXiv:math.RT/0410187.

11. Caldero, P., Chapoton, F., \& Schiffler, R. (2006). Quivers with relations arising from clusters ( $A_{n}$ case). Trans. Am. Math. Soc., 358(3), 1347-1364. arXiv:math.RT/0401316.

12. Caldero, P., Chapoton, F., \& Schiffler, R. (2006). Quivers with relations and cluster tilted algebras. Algebr. Represent. Theory, 9(4), 359-376. arXiv:math.RT/0411238.

13. Caldero, P., \& Keller, B. From triangulated categories to cluster algebras. Invent. Math., to appear. arXiv:math.RT/0506018.

14. Caldero, P., \& Keller, B. From triangulated categories to cluster algebras II. Ann. Sci. École Norm. Sup., to appear. arXiv:math.RT/0510251.

15. Fomin, S., \& Zelevinsky, A. (2002). Cluster algebras I. Foundations. J. Am. Math. Soc., 15(2), 497-529 (electronic). arXiv:math.RT/0104151.

16. Fomin, S., \& Zelevinsky, A. (2003). Cluster algebras II. Finite type classification. Invent. Math., 154(1), 63-121. arXiv:math.RA/0208229. 
17. Fomin, S., Shapiro, M., \& Thurston, D. (2006). Cluster algebras and triangulated surfaces. Part I: Cluster complexes. Preprint. arXiv:math.RA/0608367.

18. Gabriel, P. (1972). Unzerlegbare Darstellungen. Manuscripta Math., 6, 71-103.

19. Gabriel, P. (1980). Auslander-Reiten sequences and representation-finite algebras. In Representation theory, I. Proc. Workshop, Carleton Univ., Ottawa, ON, 1979. Lecture notes in math. (Vol. 831, pp. 1-71). Berlin: Springer.

20. Happel, D. (1988). Triangulated categories in the representation theory of finite dimensional algebras. London Mathematical Society, lecture notes series, Vol. 119. Cambridge: Cambridge University Press.

21. Keller, B. (2005). On triangulated orbit categories. Doc. Math., 10, 551-581 (electronic). arXiv:math.RT/0503240.

22. Keller, B., \& Reiten, I. (2005). Cluster-tilted algebras are Gorenstein and stably Calabi-Yau. Preprint. arXiv:math.RT/0512471.

23. Koenig, S., \& Zhu, B. (2006). From triangulated categories to abelian categories-cluster tilting in a general framework. Preprint. arXiv:math.RT/0605100.

24. Riedtmann, C. (1990). Algebren, Darstellungsköcher, Überlagerungen und zurück. Comment. Math. Helv., 55(2), 199-224.

25. Ringel, C. M. (1984). Tame algebras and integral quadratic forms. Lecture notes in math., Vol. 1099. Berlin: Springer.

26. Zhu, B. (2006). Equivalences between cluster categories. J. Algebra, 304, 832-850. arXiv:math.RT/0511382. 